\title{
Comportamento de risco para transtorno alimentar em universitárias brasileiras
}

\author{
Eating disorders risk behavior in Brazilian female university students
}

\author{
Marle dos Santos Alvarenga1, Fernanda Baeza Scagliusi², Sonia Tucunduva Philippi ${ }^{3}$ \\ 1 Departamento de Nutrição da Faculdade de Saúde Pública da Universidade de São Paulo (FSP-USP), Ambulatório de Bulimia e Transtornos Alimentares do Instituto de Psiquiatria do Hospital das \\ Clínicas da Faculdade de Medicina da USP (Ambulim, IPq-HC-FMUSP), Departamento de Psiquiatria, IPq, USP, São Paulo, Brasil. \\ 2 Universidade Federal de São Paulo (Unifesp) - Campus Baixada Santista, São Paulo, Brasil. \\ ${ }^{3}$ Departamento de Nutrição da Faculdade de Saúde Pública da USP.
}

Recebido: 6/1/2010 - Aceito: 10/3/2010

\begin{abstract}
Resumo
Contexto: Comportamentos de risco para transtornos alimentares envolvem atitudes e práticas inadequadas para com o alimento e o peso e podem ser avaliados com base em instrumentos validados. Objetivos: Avaliar comportamento de risco para transtornos alimentares em universitárias brasileiras das cinco regiões do país. Métodos: 2.483 universitárias responderam ao Teste de Atitudes Alimentares (EAT-26) nas cinco regiões. A pontuação no teste foi comparada entre as regiões por meio do teste qui-quadrado. Possíveis associações ou correlações com curso de graduação, idade, estado nutricional, renda individual e escolaridade do chefe da família foram avaliadas pelos coeficientes de Pearson e Spearman. Uma análise de covariância comparou o escore do EAT entre as regiões. Resultados: A frequência de comportamento de risco para transtornos alimentares variou de $23,7 \%$ a $30,1 \%$ nas cinco regiões e não houve diferença na pontuação média do EAT e na proporção de escores positivos para comportamento de risco entre as regiões. Não houve forte correlação do escore do EAT com nenhuma das variáveis. Conclusão: Universitárias brasileiras apresentam alta frequência de comportamentos de risco para TA em todas as regiões do país. Medidas de prevenção devem ser planejadas para a população jovem feminina do Brasil.
\end{abstract}

Alvarenga MS, et al. / Rev Psiq Clín. 2011;38(1):3-7

Palavras-chave: Transtornos alimentares, EAT-26, fatores de risco, atitudes alimentares, universitárias.

\begin{abstract}
Background: Risk behavior for eating disorders enrolled inadequate practices and attitudes with food and weight control and could be evaluated with valid instruments. Objectives: To evaluate risk behaviors for eating disorders among Brazilian female university students from different regions. Methods: 2.483 college students filled out the Eating Attitudes Test (EAT-26) at five regions in Brazil. The test score was compared among regions by chi-square test and possible associations or correlations with major, age, nutritional status, individual income and parental education evaluated by Pearson and Spearman coefficients. A covariance analysis compared the EAT scores among regions. Results: The frequency of positive eating disorder behavior ranges from $23.7 \%$ to $30.1 \%$ among five regions. There was no difference in EAT scores among regions, and there was not strong correlation between EAT scores and the variables studied. Discussion: Brazilian female university students have high frequency of risk behaviors for eating disorders in all regions of the country. Prevention strategies must be developed for the young female population of Brazil.
\end{abstract}

Alvarenga MS, et al. / Rev Psiq Clín. 2011;38(1):3-7

Keywords: Eating disorders, EAT-26, risk factors, eating attitudes, college students.

\section{Introdução}

Transtornos alimentares (TA) são distúrbios psiquiátricos de etiologia multifatorial caracterizados por consumo, padrões e atitudes alimentares extremamente perturbadas e excessiva preocupação com o peso e a forma corporal ${ }^{1,2}$. O diagnóstico de um TA tem critérios estabelecidos pela Organização Mundial de Saúde, no Código Internacional de Doenças ${ }^{3}$, e pela Associação de Psiquiatria Americana, no Manual de Estatísticas de Doenças Mentais (DSM-IV)1, e deve ser feito preferencialmente por um psiquiatra. A baixa prevalência e incidência dos TA torna os estudos de prevalência ou incidência de difícil realização. A utilização de entrevistas estruturadas feitas por psiquiatra para diagnóstico é inviável em grandes estudos, assim instrumentos de avaliação - como questionários - foram desenvolvidos tendo a vantagem da objetividade, baixo custo e possibilidade de exploração estatística ${ }^{4}$. Estudos epidemiológicos com TA são complexos e difíceis de serem realizados, portanto pesquisas sobre comportamento de risco podem dar um indício do problema em determinado local e população $0^{4,5}$.
As estimativas de incidência e prevalência de TA variam muito dependendo da amostragem e métodos de avaliação; sugere-se que a prevalência de bulimia nervosa $(\mathrm{BN})$ tenha tido leve decréscimo, enquanto a prevalência de anorexia nervosa ( $\mathrm{AN}$ ) parece ter tido leve acréscimo ${ }^{2}$. Aponta-se que a maior frequência de TA está entre mulheres jovens, afetando 3,2\% daquelas entre 18 e 30 anos $^{6}$. As taxas de prevalência de TA, dependendo do quadro - síndromes completas ou parciais -, variam de $0,5 \%$ a $5 \% 5$. Mesmo indivíduos com transtornos alimentares não especificados - considerados menos graves - têm risco de morte elevado ${ }^{7}$ e sabe-se que os TA têm alto custo para o sistema de saúde 8 .

Os comportamentos de risco precedem os TA clássicos e entre os fatores envolvidos estão o gênero e a etnicidade ${ }^{6}$. Os comportamentos de risco tiveram aumento de incidência e são frequentes em estudantes universitárias ${ }^{2}$. A problemática dos TA é global e a prevalência de pessoas com sintomas é considerável. Estudos internacionais que avaliaram comportamento de risco encontraram frequências variando de 6,2\% na China ${ }^{9}$ a 21,7\% no Paquistão ${ }^{10}$. 
No Brasil, os estudos que avaliaram comportamento de risco também encontraram prevalências preocupantes. Ferreira e Veiga ${ }^{11}$, avaliando uma população de adolescentes de 12 a 19 anos, com baixo nível socioeconômico, no Rio de Janeiro, encontraram 37,3\% com sintomas de compulsão alimentar e $24,7 \%$ fazendo dieta restritiva. O único estudo nacional de base populacional é o de Nunes et al. ${ }^{12}$, que avaliou uma amostra representativa de mulheres de Porto Alegre por meio do Teste de Atitudes Alimentares (EAT-26) e do Teste de Investigação Bulímica de Edinburgo (BITE), encontrando 16,5\% da população com sintomas de TA, segundo o EAT-26, e 2,9\%, segundo o BITE. Avaliando 1.807 crianças e adolescentes de 7 a 19 anos, em Minas Gerais, Vilela et al. ${ }^{13}$ encontraram 13,3\% com sintomas de TA, segundo o EAT-26, e 1,1\%, segundo o BITE.

Os estudos nacionais sobre comportamento de risco para TA no Brasil foram localizados e realizados com amostras pequenas e variadas - como escolares, adolescentes e estudantes universitárias ${ }^{13-18}$. Nenhum estudo foi realizado até o momento com um mesmo grupo etário, em diferentes localidades do Brasil, a fim de investigar diferenças e/ou semelhanças entre a frequência dos comportamentos de risco para TA.

Sabe-se que os TA têm forte componente cultural $^{2}$ e, como o Brasil tem dimensões continentais e grande diversidade cultural, é possível supor que tais fatores podem alterar a prevalência de TA em cada região. Essa hipótese nunca foi testada no Brasil previamente. O presente trabalho objetivou, portanto, investigar possíveis diferenças no comportamento de risco para TA em universitárias das cinco regiões do Brasil e avaliar possíveis associações e correlações com o curso de graduação, estado nutricional, faixa etária, renda individual e escolaridade do chefe da família.

\section{Métodos}

Local

A avaliação de comportamento de risco para TA em universitárias de diferentes regiões do Brasil esteve inserida dentro de projeto de pesquisa de avaliação de atitudes alimentares utilizando outro instrumento validado para esse fim e envolveu a parceria com instituições de ensino superior, públicas e privadas no Brasili19.

Uma amostra de estudantes universitárias foi definida para avaliar mulheres jovens em um estudo transversal. O tamanho da amostra foi determinado mediante cálculos feitos pelo OpenEpi software (Dean AG, Sullivan KM \& Soe MM, Rollins School of Public Health - Emory University, Atlanta, GA, USA. Updated 2007), considerando: a) poder, de $80 \%$; b) significância, de $5 \%$; c) o total da população de mulheres universitárias no Brasil em 2005 de acordo com o Ministério da Educação (2.488.927); e d) a frequência hipotética do fator de resultado nessa população, de $8,3 \%{ }^{16}$. Utilizando-se esses parâmetros, verificou-se que seria necessária uma amostra regional de 117 sujeitos.

O fator utilizado foi risco para TA avaliado pelo EAT-2620. $\mathrm{O}$ resultado encontrado por Feio ${ }^{16}$ foi escolhido porque este trabalho foi realizado com estudantes universitárias numa amostra randomizada, enquanto outros realizados no Brasil com população universitária foram apenas feitos com estudantes de cursos específicos - Nutrição ou Medicina, por exemplo ${ }^{17,18,21}$.

Foram enviados convites oficiais a 130 instituições de ensino superior das cinco regiões do Brasil por e-mail, utilizando-se a lista do Conselho Federal de Nutrição de 2007, convidando para parceria em pesquisa; 37 delas aceitaram participar.

\section{Sujeitos}

Foram definidos para pesquisa os cursos de Enfermagem e/ou Psicologia prioritariamente, por serem cursos eminentemente femininos. Não havendo esses cursos na instituição, foram indicados os de Fonoaudiologia, Fisioterapia, Farmácia e/ou Biomedicina - por serem os mais presentes em todas as instituições de ensino na área da saúde.
Foram convidados a participar da pesquisa estudantes desses cursos universitários, dos primeiros e segundos anos do curso, para maior homogeneização da amostra. Os critérios de inclusão foram: ser do gênero feminino; ter idade maior ou igual a 18 anos e menor ou igual a 50 anos; preencher o termo de consentimento. Os critérios de exclusão foram: ser nutricionista e/ou cursar graduação em Nutrição. Embora a lista do CFN tenha sido utilizada para contato com as instituições - por conta da formação profissional dos autores -, optou-se pela não aplicação da escala aos estudantes de Nutrição, porque alguns estudos apontam que estudantes dessa área têm comportamento alimentar alterado ${ }^{17,21,22}$. Pela proximidade com a área da nutrição e corpo, não foram convidados os alunos do curso de Educação Física.

\section{Instrumento}

Os responsáveis locais receberam instruções específicas sobre a pesquisa e aplicação dos instrumentos, que deveriam ser preenchidos em sala de aula pelos participantes que respeitassem os critérios de inclusão e exclusão. As alunas responderam também dados de caracterização como idade, peso e estatura autorreferidos, renda individual e grau de instrução do chefe da sua família.

O Teste de Atitudes Alimentares utilizado para avaliação de comportamento de risco é um instrumento autoaplicável que serve para rastreamento de sintomas de TA, traduzido para o português por Nunes et al. ${ }^{23} \mathrm{e}$ depois validado por Bighetti et al. ${ }^{24}$. O teste avalia sintomas de restrição alimentar e identifica comportamentos anoréxicos e de dieta. O instrumento foi validado no Canadá com pacientes com AN e jovens universitárias saudáveis. A versão original do EAT40 era constituída de 40 itens e, mais tarde, os autores propuseram a versão abreviada com 26 questões - EAT-2620. Concluiu-se que o teste não faz diagnóstico do distúrbio, mas detecta casos clínicos em população de alto risco e identifica indivíduos com preocupações anormais com alimentação e peso. O instrumento possui seis opções de resposta, que variam de 0 a 3 pontos (sempre $=3$; muitas vezes $=2$; às vezes $=1$; poucas vezes, quase nunca e nunca $=0)$; a questão 25 tem resposta invertida. A pontuação maior ou igual a 21 no EAT identifica o indivíduo em grupo de risco para $\mathrm{TA}^{20}$.

O EAT é um questionário muito utilizado em avaliações de comportamento de risco e sintomas de TA - com pacientes e em estudos populacionais; ele é também utilizado em estudos internacionais com populações diversas, dada a sua facilidade de aplicação, eficiência, economia e não necessidade de treinamento para administração 4,25 . O EAT é provavelmente o mais usado de todos os instrumentos de autopreenchimento na área dos $\mathrm{TA}^{25}$.

A versão de Nunes et al. ${ }^{26}$ para o EAT-26 teve consistência interna (Alpha de Cronbach) de 0,75; o trabalho de Bighetti et al. ${ }^{24}$ validando outra versão em português do EAT-26 com adolescentes do sexo feminino encontrou Alpha de Cronbach de 0,82 - essa versão realizou também uma revisão das questões de cada subescala após análise fatorial e alocou a ordem das questões no teste. Como essa versão realizou análise fatorial e encontrou maior consistência interna no instrumento, optou-se por utilizá-la para aplicação nas universitárias.

\section{Análise dos dados}

As análises estatísticas foram conduzidas por meio do software SPSS 12.0 (Statistical Package for Social Science Inc., Chicago, Illinois USA). Para todas as análises foi considerado um nível de significância de 5\%.

Uma análise de correlação entre as variáveis quantitativas foi realizada utilizando o coeficiente de Pearson, e para avaliar o escore do EAT com as variáveis qualitativas foi utilizado o coeficiente de Spearman.

Foram consideradas como tendo comportamento de risco para TA as universitárias cuja pontuação do EAT-26 foi $\geq 21$ (escore positivo) e sem comportamento de risco aquelas cujo escore foi $<21$ (escore negativo). Esses resultados, como escore positivo e negativo, foram comparados por meio do teste qui-quadrado entre as regiões brasileiras. 
Uma análise de covariância comparou o escore do EAT entre as regiões utilizando como covariáveis a idade, o índice de massa corpórea (IMC), a renda mensal individual em faixas de salários mínimos, a escolaridade do chefe da família e o curso de graduação. As suposições de homocedasticidade foram verificadas para utilização desse teste.

\section{Aspectos éticos}

As universitárias preencheram o termo de consentimento livre e esclarecido. A pesquisa foi aprovada pelo Comitê de Ética da Faculdade de Saúde Pública da Universidade de São Paulo.

\section{Resultados}

Do total de estudantes que participaram da pesquisa sobre atitudes alimentares $(\mathrm{n}=2.489), 2.483$ preencheram corretamente o EAT26. Do total de participantes, $9,6 \%$ era da Região Norte, $15,4 \%$, da Região Nordeste, 8,0\%, da Região Centro-Oeste, 35,6\%, da Região Sudeste e 31,4\%, da Região Sul. Em relação ao curso de graduação, $58,7 \%$ cursavam Enfermagem, 15,1\%, Psicologia, 12,3\%, Farmácia, 9,4\%, Fisioterapia, 1,8\%, Biomedicina, 1,3\%, Fonoaudiologia e 1,4\% não informaram o curso.

Os dados de idade, peso, altura e IMC das universitárias podem ser observados na tabela 1. Quando o IMC foi classificado de acordo com a Organização Mundial de Saúde ${ }^{27}$, observou-se que a maioria (70,6\%) estava eutrófica ${ }^{19}$.

Quando classificadas de acordo com a renda mensal em faixa de salários mínimos (SM), 45\% declararam receber menos de 2 $\mathrm{SM} / \mathrm{mês} ; 26 \%$, de 2 a $4,9 \mathrm{SM} / \mathrm{mês} ; 5,9 \%$, de 5 a 9,9 SM/mês e $1,8 \%$, mais de $10 \mathrm{SM} /$ mês (1.958 universitárias forneceram esse dado $=78,7 \%)$. Quando avaliadas segundo o nível de escolaridade do chefe da família, $44,7 \%$ tinham chefe com nível superior completo ou incompleto; $30,1 \%$, curso secundário completo ou incompleto; $16,8 \%$, fundamental completo e $4,3 \%$ eram analfabetos ou haviam estudado no máximo quatro anos (2.388 universitárias forneceram esse dado $=95,9 \%)^{19}$.
Observam-se na tabela 1 os resultados do escore do EAT-26 nas diferentes regiões brasileiras. $\mathrm{Na}$ análise de covariância, observou-se que apenas o IMC influenciava no modelo final $(p=0,001)$. Com o modelo ajustado pelo IMC, o teste apontou que não houve diferença nas médias das pontuações entre as diferentes regiões $(p=0,210)$.

A correlação entre o escore do EAT-26 e as variáveis foi inexistente ou muito baixa: 0,025 para idade; 0,213 para IMC; $-0,030$ para curso; 0,001 para renda e 0,010 para escolaridade.

Quando o resultado na pontuação do EAT-26 foi avaliado segundo escore positivo (EAT+) e escore negativo (EAT-) para comportamento de risco, encontrou-se que $26,1 \%$ da amostra total apresentava comportamento de risco para um TA (Tabela 2). A maior proporção de escores positivos foi encontrada na Região Norte e a menor, na Região Centro-Oeste, porém de acordo com o teste do qui-quadrado não houve diferença estatisticamente significante entre as distribuições $(p=0,298)$.

\section{Discussão}

Encontrou-se nas universitárias brasileiras uma frequência alta e preocupante de comportamentos de risco para TA em todas as regiões do país. Até onde se sabe, esse foi o estudo com maior tamanho amostral, quando comparado às pesquisas brasileiras e internacionais. Por questões logísticas e financeiras, foi impossível o deslocamento para todas as regiões para a coleta desses dados, mas, mesmo sem trabalhar com uma amostra probabilística representativa de cada região, acredita-se que, pelo número expressivo de universitárias participantes e pela inexistência de trabalhos comparativos em diferentes regiões do Brasil, esses resultados ilustram a realidade dos comportamentos de risco para TA em universitárias brasileiras.

O EAT-26, assim como outros instrumentos de avaliação, não faz diagnóstico de transtornos alimentares e então não se pode afirmar sobre quadros de TA nessa população, mas comportamentos de risco são apontados. Uma nomenclatura sugerida da Associação Dietética Americana e utilizada em diferentes trabalhos é o comer transtornado (do inglês disorderd eating), caracterizado por comportamentos alimentares problemáticos e métodos inadequados para perder ou

Tabela 1. Idade, peso, altura, índice de massa corpórea (IMC) e pontuação no Teste de Atitudes Alimentares (EAT-26) da amostra de universitárias das diferentes regiões do Brasil

\begin{tabular}{|c|c|c|c|c|c|c|c|}
\hline Região & Norte (N) & Nordeste (NE) & Centro-oeste (CO) & Sudeste (SE) & Sul (S) & Total nacional & $P$ \\
\hline Idade & $\begin{array}{c}26,1 \pm 7,1(24,0) a \\
n \pm 231\end{array}$ & $\begin{array}{c}24,4 \pm 6,5(23,0)^{b} \\
n=376\end{array}$ & $21,9 \pm 5,4(20) \wedge n=193$ & $\begin{array}{c}23,1 \pm 6,1 \\
(21) \mathrm{d} n=848\end{array}$ & $\begin{array}{c}23,0 \pm 5,3(21) \mathrm{e} \\
n=776\end{array}$ & $\begin{array}{c}23,5 \pm 6,1(21) \\
n=2424\end{array}$ & 0,001 \\
\hline Peso & $\begin{array}{c}58,5 \pm 10,5(57,0) \\
n=227\end{array}$ & $\begin{array}{c}57,1 \pm 9,7(56,0)^{\mathrm{f}} \\
\mathrm{n}=373\end{array}$ & $\begin{array}{c}57,2 \pm 9,0(56,0) \\
n=195\end{array}$ & $\begin{array}{c}59,2 \pm 10,2(58,0) 9 \\
n=856\end{array}$ & $\begin{array}{c}59,0 \pm 9,9(57,0) \\
n=764\end{array}$ & $\begin{array}{c}58,6 \pm 10,0(57,0) \\
n=2415\end{array}$ & 0,002 \\
\hline Altura & $\begin{array}{c}1,61 \pm 0,1(1,60)^{n} \\
n=214\end{array}$ & $\begin{array}{c}1,62 \pm 0,1(1,61) i \\
n=368\end{array}$ & $\begin{array}{c}1,63 \pm 0,1(1,63) \\
n=191\end{array}$ & $\begin{array}{c}1,64 \pm 0,1(1,63)^{k} \\
n=852\end{array}$ & $\begin{array}{c}1,64 \pm 0,1(1,64) ! \\
n=759\end{array}$ & $\begin{array}{c}1,63 \pm 0,1(1,63) \\
n=2384\end{array}$ & 0,000 \\
\hline Imc & $\begin{array}{c}22,7 \pm 3,5(22,1)^{\mathrm{m}} \\
\mathrm{n}=211\end{array}$ & $\begin{array}{c}21,9 \pm 3,4(21,4) \\
n=364\end{array}$ & $\begin{array}{c}21,5 \pm 3,1(21,1)^{n} \\
n=191\end{array}$ & $\begin{array}{c}22,2 \pm 3,7(21,4) \\
n=841\end{array}$ & $\begin{array}{c}21,9 \pm 3,3(21,2) \\
n=750\end{array}$ & $\begin{array}{c}22,0 \pm 3,5(21,4) \\
n=2357\end{array}$ & 0,005 \\
\hline EAT-26 & $\begin{array}{c}16,5 \pm 10,8(14,0) \\
n=239\end{array}$ & $\begin{array}{c}15,8 \pm 9,8(14,0) \\
n=382\end{array}$ & $\begin{array}{c}14,3 \pm 10,0(11,5) n \\
=198\end{array}$ & $\begin{array}{c}15,5 \pm 10,5(13,0) \\
n=884\end{array}$ & $\begin{array}{c}15,0 \pm 10,8(12,0) \\
n=780\end{array}$ & $\begin{array}{c}15,4 \pm 10,4(13,0) \\
n=2483\end{array}$ & 0,210 \\
\hline
\end{tabular}

Dados apresentados em média +/- desvio-padrão (mediana). Nota: o número de respostas foi variável por item e região e está indicado em cada um deles (n).

a: diferente do NE, CO, SE e S; b: diferente do N, CO, SE e S; c: diferente do NE e N; d: diferente do NE e N; e: diferente do NE e N; f: diferente do SE; g: diferente do NE; h: diferente do CO, SE e S; i: diferente do CO, SE e S; j: diferente do NE e N; k: diferente do NE e N; I: diferente do NE e N; m: diferente do CO; n: diferente do N.

Tabela 2. Comportamento de risco para transtorno alimentar segundo pontuação no Teste de Atitudes Alimentares (EAT-26) da amostra de universitárias nas diferentes regiões do Brasil

\begin{tabular}{|c|c|c|c|c|c|c|c|c|c|c|c|c|c|}
\hline \multirow{3}{*}{ EAT-26 } & \multirow{2}{*}{\multicolumn{2}{|c|}{ Amostra Total }} & \multicolumn{10}{|c|}{ Distribuição por Região } & \\
\hline & & & \multicolumn{2}{|c|}{ Norte } & \multicolumn{2}{|c|}{ Nordeste } & \multicolumn{2}{|c|}{ Centro-0este } & \multicolumn{2}{|c|}{ Sudeste } & \multicolumn{2}{|c|}{ Sul } & \\
\hline & $\mathrm{n}$ & $\%$ & $\mathrm{n}$ & $\%$ & $\mathrm{n}$ & $\%$ & $\mathrm{n}$ & $\%$ & $\mathrm{n}$ & $\%$ & $\mathrm{n}$ & $\%$ & \\
\hline Comportamento de risco positivoa & 648 & 26,1 & 72 & 30,1 & 110 & 28,8 & 47 & 23,7 & 226 & 25,6 & 193 & 24,7 & \multirow[t]{2}{*}{$p=0,298$} \\
\hline Comportamento de risco negativo ${ }^{b}$ & 1835 & 73,9 & 167 & 69,9 & 272 & 71,2 & 151 & 76,3 & 658 & 74,4 & 587 & 75,3 & \\
\hline $\mathrm{N}$ válido & \multicolumn{2}{|c|}{2483} & \multicolumn{2}{|c|}{239} & \multicolumn{2}{|c|}{382} & \multicolumn{2}{|c|}{198} & \multicolumn{2}{|c|}{884} & \multicolumn{2}{|c|}{780} & \\
\hline
\end{tabular}

a: escore $\geq 21$; b: escore $<21$ 
controlar o peso que ocorrem com menor frequência e de forma menos severa do que o exigido pelos critérios diagnósticos ${ }^{6}$.

O percentual de EAT positivo para comportamento de risco foi maior do que o encontrado em outros estudos com universitários no país. Souza et al. ${ }^{18}$ encontraram 5,5\% de EAT+ em alunas do curso de Medicina avaliadas na Universidade Federal do Ceará (UFC). Bosi et al.14,28,29 avaliaram alunas de Nutrição, Psicologia e Educação Física no Rio de Janeiro e encontraram 14,0\%, 6,9\% e 6,9\%, respectivamente, com EAT+. Feio ${ }^{16}$ encontrou $8,3 \%$ de EAT+ na avaliação de universitárias da Universidade Federal de Santa Catarina (UFSC).

$O$ resultado desta pesquisa foi similar apenas ao encontrado por Fiates e Salles ${ }^{17}$, avaliando alunas da UFSC, em que o percentual de EAT+ foi de $22,2 \%$, e no grupo de alunas do curso de Nutrição, o percentual foi maior que nos outros grupos (25,4\% na Nutrição e $18,7 \%$ nos demais). Antonaccio ${ }^{21}$ também encontrou valores maiores de EAT+ em estudantes do curso de Nutrição da Universidade de São Paulo (USP) e de uma instituição particular em São Paulo, e a média foi de $40 \%$ de EAT+, variando de $27,8 \%$ a $50 \%$, dependendo do período do curso em andamento.

Uma possível consideração para os maiores valores encontrados no presente trabalho pode ser devida à versão do EAT utilizada, que foi a de Bighetti et al. ${ }^{24}$, e não a versão de Nunes et al. ${ }^{23}$ que foi a utilizada nos demais trabalhos nacionais; esta última versão, no entanto, mostrou baixos coeficientes de validade ${ }^{26}$. Em seu trabalho com adolescentes, Bighetti et al. ${ }^{24}$ encontraram 41,6\% com EAT+, com a mesma versão do EAT-26 utilizada no presente trabalho. Outro possível fator de diferença é o uso do ponto de corte de 20 ou 21 para EAT+ nos diferentes trabalhos.

Trabalhos internacionais realizados com estudantes universitárias também encontram resultados diversos. Nos Estados Unidos (EUA), Anstine e Grinenko ${ }^{30}$ encontraram 17,0\% de EAT+ em universitárias na Flórida; Hoerr et al ${ }^{31}$ encontraram 10,9\% de EAT+ em universitárias de Michigan; e Nelson et al. ${ }^{32}$ encontraram 20\% com de EAT+ em universitárias do Meio-Oeste Americano. Em diferentes localidades americanas, outros estudos encontraram valores de EAT+ para universitárias de $11,0 \%{ }^{33}, 17,0 \%^{34}$ e $19,1 \%{ }^{35}$.

O EAT já foi traduzido para diversas línguas - seja na sua versão de 40 ou de 26 questões -, entre elas japonês, alemão, francês e irdu (no Paquistão). Muitos dos estudos realizados em países fora da América utilizaram o EAT-40 ou foram realizados com adolescentes (a população mais avaliada) e estudantes de segundo grau. Em culturas diferentes da americana, os valores de EAT-26+ em estudos com universitárias são também variados. Makino et al. ${ }^{9}$, avaliando universitárias em Pequim na China, encontraram 6,2\% com EAT+. Ambrosi-Randic e Pokrajac-Bulian ${ }^{36}$ encontraram 11,3\% de EAT+ em universitárias na Croácia. Celikel et al. ${ }^{37}$ encontraram $12 \%$ de uma amostra de estudantes universitárias da Turquia com EAT+. Babar et al. ${ }^{10}$, avaliando estudantes de Medicina e Enfermagem em Karachi no Paquistão, encontraram um total de $21,7 \%$ de EAT+. Avaliando também estudantes de Medicina na Cracóvia, Kolarzyk e Jaglarz ${ }^{38}$ encontraram $8,9 \%$ de comportamento de risco para TA segundo EAT-26. Em outro estudo no Paquistão, desta vez com mulheres que haviam terminado a faculdade, mas de idade muito semelhante à do grupo de universitárias (18-24 anos), Suhail e Zaib-u-Nisa ${ }^{39}$ encontraram $17,0 \%$ de EAT+.

Pode-se perceber que a presente pesquisa encontrou valores superiores de EAT+ quando se compara aos estudos americanos e internacionais com universitárias. Não é possível afirmar, no entanto, que a frequência de comer transtornado ou comportamentos de risco para TA sejam maiores em universitárias brasileiras do que de outros países, dadas as diferenças de amostragem, metodologia, objetivo dos estudos e mesmo de outras características (faixa etária, renda, cultura, situação social) das estudantes avaliadas. De qualquer forma, os valores encontrados nesta amostra de universitárias chamam atenção pela magnitude da presença de comportamentos de risco.

O fato de diferenças estatísticas não terem sido encontradas entre as regiões pode apontar limitações da amostragem desta pesquisa; também se pode pensar que o EAT não é um bom instrumento para captar diferenças culturais. Deve-se ainda considerar que fatores pessoais como experiências alimentares e cultura familiar podem ter maior peso no comportamento de risco do que o curso da universitária, seu estado nutricional, renda e escolaridade do chefe da família. Outra questão a ser considerada é a presença de uma globalização de informações sobre beleza, magreza e dietas no país e a "pressão" com relação a elas e que, portanto, diferentes grupos, mesmo de estágio de vida e classe social semelhantes (como estudantes universitárias), não apresentem respostas diferentes ao teste; além disso, uma diferença na frequência de comportamentos de risco na verdade não exista nessa população.

Não ter encontrado forte correlação com as variáveis estudadas aponta que outras variáveis - como a insatisfação corporal e a influência da mídia - devam ser avaliadas em relação aos comportamentos de risco para TA. Estudo de Cenci et al. ${ }^{40}$ avaliando comportamento bulímico de universitárias brasileiras em Florianópolis encontrou que a insatisfação com a imagem corporal foi o mais importante fator associado e que não houve correlação de sintomas bulímicos com a renda familiar ou escolaridade da mãe. Segundo Andrade et al. ${ }^{41}$, os TA são prevalentes em adolescentes e adultos jovens pertencentes a todos os grupos étnicos, e não apenas entre indivíduos de cor branca e de países industrializados, e que há uma discordância de que os TA sejam um fenômeno puramente "ocidental", com estudos apontando para uma distribuição socioeconômica mais ampla dos TA.

De qualquer modo, a magnitude dos comportamentos de risco apontados por este trabalho é importante, uma vez que esses comportamentos podem evoluir para síndromes completas de TA, além de levarem a outros prejuízos à saúde e a consequências psicossociais. Assim como no caso da obesidade, a prevenção dos TA tem sido colocada como de emergência em saúde ${ }^{42,43}$, uma vez que há aumento da demanda, opções limitadas de tratamento, os casos são crônicos com recaídas frequentes e o tratamento do distúrbio é um dos mais caros entre todos os quadros psiquiátricos.

A revisão sistemática de Simon et al. ${ }^{8}$ apontou que no Reino Unido o custo com tratamento da AN em 1990 foi de 4,2 milhões de libras; na Alemanha, o custo foi de 65 milhões de euros para AN e 10 milhões de euros para BN e na Austrália o custo com tratamento dos TA em 1993/1994 foi de 22 milhões de dólares australianos. Não há estudo sobre custo dos TA no Brasil, de qualquer forma a presença dos comportamentos de risco deve ser considerada por profissionais e pelo sistema de saúde visando à adequada detecção, prevenção e tratamento dos casos.

No tocante a ações de saúde pública, as medidas de prevenção devem ser articuladas em vários níveis, envolvendo, por exemplo, a formação dos profissionais de atenção básica para o reconhecimento de sinais e sintomas de TA e a criação de programas específicos de prevenção para grupos de risco - como o detectado nesta pesquisa.

Sugere-se, ainda, que discussões sociais sobre os fatores de risco ambientais e, portanto, passíveis de mudança, como a questão dos padrões de beleza e da prática de dietas, devam ocorrer em vários setores, como mídia, indústria da moda, área da saúde e da educação. Profissionais de saúde devem estar atentos a sinais de risco para TA, e não apenas aos quadros completos; e escolas e universidades deveriam planejar a abordagem desses temas em seus programas educacionais.

Pode-se concluir que o comportamento de risco para TA é frequente entre universitárias brasileiras. Estes resultados apontam para uma relação inadequada com o alimento e com o corpo, que pode ter consequências físicas e psíquicas e que deve ser considerada por profissionais de saúde e educadores para planejamento de estratégias de educação nutricional e programas de prevenção de transtornos alimentares.

\section{Agradecimentos}

Agradecemos à Fundação de Amparo à Pesquisa do Estado de São Paulo (Fapesp) pelo auxílio à realização deste trabalho (processo 06/56850-9). Também agradecemos aos coordenadores do curso de Nutrição e seus parceiros pela expressiva colaboração na coleta de dados nas instituições participantes: Centro de Ensino Unificado de Teresina, Centro Universitário de Barra Mansa, Centro Universitário de Brasília, Centro Universitário de Vila Velha, Centro Universitário 
de Volta Redonda, Centro Universitário do Sul de Minas, Centro Universitário Feevale, Centro Universitário La Salle, Centro Universitário Nilton Lins, Faculdade Itabirana de Saúde, Faculdade Natalense para o Desenvolvimento do Rio Grande do Norte, Faculdade São Lucas Faculdade Vale do Ipojuca, Pontifícia Universidade Católica do Paraná, Unidade de Ensino Superior do Sul do Maranhão, Universidade Católica Dom Bosco, Universidade Cruzeiro do Sul, Universidade de Brasília, Universidade de Caxias do Sul, Universidade de Cuiabá, Universidade do Sul de Santa Catarina, Universidade do Vale do Itajaí, Universidade do Vale do Rio dos Sinos, Universidade Federal de Alfenas, Universidade Federal de Pelotas, Universidade Federal de Santa Catarina, Universidade Federal do Mato Grosso, Universidade Federal do Paraná, Universidade Federal do Piauí, Universidade Federal do Rio Grande do Sul, Universidade Metodista de Piracicaba, Universidade Metodista de São Paulo, Universidade Municipal de São Caetano do Sul, Universidade Paulista Campus Manaus/São Paulo-Capital/Sorocaba.

\section{Referências}

1. American Psychiatry Association (APA). Diagnostic and Statistical Manual of Mental Disorders (DSM-IV). 4. ed. Washington DC: American Psychiatry Association; 1994.

2. American Psychiatry Association (APA). Practice guideline for the treatment of patients with eating disorders. 3. ed. Virginia: American Psychiatric Publishing; 2006.

3. Organização Mundial de Saúde. Classificação de transtornos mentais e de comportamento da CID-10. Descrições clínicas e diretrizes diagnósticas. Porto Alegre: Artes Médicas; 1993.

4. Freitas S, Gorenstein C, Appolinario JC. Instrumentos para a avaliação dos transtornos alimentares. Rev Bras Psiquiatr. 2002;24:34-8.

5. Hay PJ. Epidemiologia dos transtornos alimentares: estado atual e desenvolvimentos futuros. Rev Bras Psiquiatr. 2002;24:13-7.

6. American Dietetic Association (ADA). Position of the American Dietetic Association: nutrition intervention in the treatment of anorexia nervosa, bulimia nervosa, and other eating disorders. J Am Diet Assoc. 2006;106:2073-82.

7. Crow SJ, Peterson CB, Swanson SA, Raymond NC, Specker S, Eckert ED, et al. Increased mortality in bulimia nervosa and other eating disorders. Am J Psychiatry. 2009;166:1342-6.

8. Simon J, Schmidt U, Pilling S. The health service use and cost of eating disorders. Psychol Med. 2005;35:1543-51.

9. Makino M, Hashizume M, Tsuboi K, Yasushi M, Dennerstein L. Comparative study of attitudes to eating between male and female students in the People's Republic of China. Eat Weight Disord. 2006;11:111-7.

10. Babar N, Alam M, Ali SS, Ansari A, Atiq M, Awais A, et al. Anorexic behaviour and attitudes among female medical and nursing students at a private university hospital. J Pak Med Assoc. 2002;52:272-6.

11. Ferreira JES, Veiga GV. Eating disorder risk behavior in Brazilian adolescents from low socio-economic level. Appetite. 2008;51:249-55.

12. Nunes MA, Barros FC, Olinto MTA, Camey S, Mari JDJ. Prevalence of abnormal eating behaviors and inappropriate methods for weight control in young women from Brazil: a population - based study. Eat Weight Disord. 2003;8:100-6.

13. Vilella JEM, Lamounier JA, Dellaretti Filho MA, Neto JRB, Horta GM. Transtornos alimentares em escolares. J Pediatr. 2004;80:49-54.

14. Bosi MLM, Luiz RR, Morgado CMC, Costa MLS, Carvalho RJ. Autopercepção da imagem corporal entre estudantes de nutrição no Rio de Janeiro. J Bras Psiquiatr. 2006;55:34-40.

15. Dunker KLL, Philippi ST. Differences in the diet composition of Brazilian adolescent girls with positive or negative score in the Eating Attitudes Test. Eat Weight Disord. 2005;10:e70-5.

16. Feio LC. Prevalência de sintomas de anorexia nervosa e insatisfação com a imagem corporal em universitárias ingressantes na Universidade Federal de Santa Catarina. [dissertação] Santa Catarina: Universidade Federal de Santa Catarina; 2007.

17. Fiates GMR, Salles RK. Fatores de risco para o desenvolvimento de distúrbios alimentares: um estudo em universitárias. Rev Nutr. 2001;14:3-6.
18. Souza FGM, Martins MCR, Monteiro FCC, Neto GCM, Ribeiro IB. Anorexia e bulimia nervosa em alunas da Faculdade de Medicina da Universidade Federal do Ceará. Rev Psiquiatr Clín. 2002;24:172-80.

19. Alvarenga MS, Scagliusi FB, Philippi ST. Comparison of food attitudes among college students from the five Brazilian regions. Ciênc Saúde Coletiva. 2010; in press.

20. Garner DM, Olmested MP, Bohr Y, Garfinkel PE. The eating attitude test: psychometric features and clinical correlates. Psychol Med. 1982;12:871-79.

21. Antonaccio CMA. Estudantes de nutrição: uma ótica sobre o comportamento alimentar e os transtornos alimentares [dissertação]. São Paulo: FCF-FEA-FSP/USP; 2001.

22. Fredenberg JP, Berglund PT, Dieken HA. Incidence of eating disorders among selected female university students. J Am Diet Assoc. 1996;96:64-8.

23. Nunes MA, Bagatini L, Abuchaim AL, Kunz A, Ramos D, Silva JA, et al. Considerações sobre o Teste de Atitudes Alimentares (EAT-26). Rev ABP-APAL. 1994;16:7-10.

24. Bighetti F, Santos CB, Santos JE, Ribeiro RPP. Tradução e validação do Eating Attitudes Test em adolescentes do sexo feminino de Ribeirão Preto-SP. J Bras Psiquiatr. 2004;53:339-46.

25. Mintz LB, O'Halloran MS. The Eating Attitudes Test: validation with DSM-IV Eating Disorders Criteria. J Pers Assess. 2000;74:489-503.

26. Nunes MA, Camey S, Olinto MTA, Mari JJ. The validity and 4-year test-retest reliability of the Brazilian version of the Eating Attitudes Test-26. Braz J Med Biol Res. 2005;38:1655-62.

27. World Health Organization (WHO). Obesity: preventing and managing the global epidemic. Report of a World Health Organization consultation on obesity. Genebra: WHO; 1998.

28. Bosi MLM, Luiz RR, Uchimura KY, Oliveira FP. Comportamento alimentar e imagem corporal entre estudantes de educação física. J Bras Psiquiatr. 2008;57:28-33.

29. Bosi MLM, Uchimura KY, Ronir Raggio Luiz RR. Eating behavior and body image among psychology students. J Bras Psiquiatr. 2009;58:150-5.

30. Anstine D, Grinenko D. Rapid screening for disordered eating in college-aged females in the primary care setting. J Adolesc Health. 2000;26:338-42.

31. Hoerr SL, Bokram R, Lugo B, Bivins T, Keast DR. Risk for disordered eating relates to both gender and ethnicity for college students. J Am Coll Nutr. 2002;21:307-14.

32. Nelson WL, Hughes HM, Katz B, Searight HR. Anorexic eating attitudes and behaviors of male and female college students. Adolescence. 1999;34:621-33.

33. Thome J, Espelage DL. Relations among exercise, coping, disordered eating and psychological health among college students. Eat Behav. 2004;5:337-51.

34. Prouty AM, Protinsky HO, Canady D. College women: eating behaviors and help-seeking preferences. Adolescence. 2002;37:353-63.

35. Anderson DA, Simmons AM, Martens MP, Ferrier AG, Sheehy MJ. The relationship between disordered eating behavior and drinking motives in college-age women. Eat Behav. 2006;7:419-22.

36. Ambrosi-Randic N, Pokrajac-Bulian A. Psychometric properties of the eating attitudes test and children's eating attitudes test in Croatia. Eat Weight Disord. 2005;10:e76-82.

37. Celikel FC, Cumurcu BE, Koc M, Etikan I, Yucel B. Psychologic correlates of eating attitudes in Turkish female college students. Compr Psychiatry. 2008;49:188-94.

38. Kolarzyk E, Jaglarz M. Zaburzone zachowania zywieniowe studentów kierunków medycznych Uniwersytetu Jagiellonskiego. [Disordered eating attitudes in medical students of Jagiellonian University]. Przegl Lek. 2003;60:48-52.

39. Suhail K, Zaib-u-Nisa. Prevalence of eating disorders in Pakistan: relationship with depresión and body shape. Eat Weight Disord. 2002;7:131-38.

40. Cenci M, Peres KG, Vasconcelos FAG. Prevalência de comportamento bulímico e fatores associados em universitárias. Rev Psiquiatr Clín. 2009;83-8.

41. Andrade LHSG, Viana MC, Silveira CM. Epidemiologia dos transtornos psiquiátricos da mulher. Rev Psiquiatr Clín. 2006;33:43-54.

42. Irving LM, Neumark-Sztainer D. Integrating the prevention of eating disorders and obesity: feasible or futile? Prev Med. 2002;34:299-309.

43. Neumark-Sztainer D. Can we simultaneously work toward the prevention of obesity and eating disorders in children and adolescents. Int J Eat Disorder. 2005;38:220-7. 